Pacific Journal of Mathematic 


\title{
ON EULER'S CRITERION FOR QUINTIC NONRESIDUES
}

\author{
Kenneth S. Williams
}

Let $p$ be a prime $\equiv 1(\bmod 5)$. If 2 is a quintic nonresidue $(\bmod p)$ then $2^{p-1 / s} \equiv \alpha(\bmod p)$ for some fifth root of unity $\alpha_{5}(\not \equiv 1)(\bmod p)$. Emma Lehmer has given an explicit expression for $\alpha_{5}$ in terms of a particular solution of a certain quadratic partition of $p$. In this paper we show how in principle the corresponding result can be obtained for any quintic nonresidue $D(\bmod p)$. Full details are given for $D=2,3,5$.

1. Introduction. Let $k$ be an integer $\geqq 2$ and let $p$ be a prime $\equiv 1(\bmod k)$. Euler's criterion states that $D^{(p-1) / k} \equiv 1(\bmod p)$ if and only if $D$ is a $k$ th power residue $(\bmod p)$. Thus if $D$ is not a $k$ th power residue $(\bmod p)$, for some $k$ th root of unity $\alpha_{k}(\not \equiv 1) \operatorname{moduo} p$ we have $D^{(p-1) / k} \equiv \alpha_{k}(\bmod p)$. Clearly $\alpha_{2}=-1$. For $k>2$ Emma Lehmer [3] has proposed the problem of specifying which $\alpha_{k}$ corresponds to a given $D$. For $D=2, k=3,4,5,8$, she has given explicit expressions for $\alpha_{k}$ in terms of certain quadratic partitions of $p$. Elsewhere the author [6] has given a complete treatment of the case $k=3$. In this paper we treat the case $k=5$. Full details are given for $D=2,3,5$. The method used is described in $\S 4$ and can be applied to any value of $D$ if the reader has the patience to supply the many details.

2. Two lemmas involving the domain $Z[\zeta]$. We set $\zeta=$ $\exp (2 \pi i / 5)$. If $Q$ denotes the field of rational numbers, the cyclotomic field formed by adjoining $\zeta$ to $Q$ is denoted by $Q(\zeta)$. The domain of integers of $Q(\zeta)$ is denoted by $Z[\zeta]$. Every element of $Z[\zeta]$ can be written in the form $a_{1} \zeta+a_{2} \zeta^{2}+a_{3} \zeta^{3}+a_{4} \zeta^{4}$, where $a_{1}, a_{2}, a_{3}, a_{4}$ are rational integers. The domain $Z[\zeta]$ is a unique factorization domain. The element $1-\zeta$ is a prime in $Z[\zeta]$ which divides 5 . The units of $Z[\zeta]$ are given by $\pm \zeta^{i}\left(\zeta+\zeta^{4}\right)^{j}$, where $i$ and $j$ are integers with $0 \leqq i \leqq 4$. If $\alpha$ and $\beta$ are associated nonzero elements, that is $\alpha / \beta$ is a unit, we write $\alpha \sim \beta$. The complex conjugate of an element $\alpha \in Z[\zeta]$ will be denoted by $\bar{\alpha}(\in Z[\zeta])$. We will need the following two results.

LEMMA 1. If $\alpha \in Z[\zeta]$ is such that $\alpha \neq 0(\bmod 1-\zeta)$ then $\alpha$ possesses an associate $\alpha^{\prime}$ such that $\alpha^{\prime} \equiv-1\left(\bmod (1-\zeta)^{2}\right)$.

Proof. Set $\alpha=a_{1} \zeta+a_{2} \zeta^{2}+a_{3} \zeta^{3}+a_{4} \zeta^{4}, b=a_{1}+a_{2}+a_{3}+a_{4}, c=$ 
$a_{1}+2 a_{2}+3 a_{3}+4 a_{4}$. As $\alpha \neq \equiv(\bmod 1-\zeta)$ we have $b \not \equiv 0(\bmod 5)$. We define $d$ uniquely by $2^{d} b \equiv-1(\bmod 5), 0 \leqq d \leqq 3$. Then we have only to choose $\alpha^{\prime}=\zeta^{c^{d}}\left(\zeta+\zeta^{4}\right)^{d} \alpha$, as $\zeta+\zeta^{4} \equiv 2\left(\bmod (1-3)^{2}\right)$ and $\zeta^{c^{2}} \equiv b\left(\bmod (1-\zeta)^{2}\right)$.

LEMMA 2. If $\alpha, \beta \in Z[\zeta]$ are such that

(a) $\alpha \bar{\alpha}=\beta \bar{\beta}$

(b) $\alpha, \beta \neq 0(\bmod 1-\zeta)$,

(c) $\alpha \equiv \beta\left(\bmod (1-\zeta)^{2}\right)$,

(d) $\alpha \sim \beta$,

then

$$
\alpha=\beta \text {. }
$$

Proof. By (d) we have $\alpha= \pm \zeta^{i}\left(\zeta+\zeta^{4}\right)^{j} \beta$, for integers $i$ and $j$ with $0 \leqq i \leqq 4$. Thus using (a) we obtain $\alpha \bar{\alpha}=\left(\zeta+\zeta^{4}\right)^{2 j} \beta \bar{\beta}=$ $\left(\zeta+\zeta^{4}\right)^{2 j} \alpha \bar{\alpha}$. Now (b) guarantees that $\alpha \neq 0$, so that $\alpha \bar{\alpha} \neq 0$, and we must have $\left(\zeta+\zeta^{4}\right)^{2 j}=1$. As $\zeta+\zeta^{4}=\frac{1}{2}(\sqrt{5}-1)>0$ we have $j=0$ and so $\alpha= \pm \zeta^{i} \beta, 0 \leqq i \leqq 4$. From (b) and (c) we have $\left( \pm \zeta^{i}-1\right) \beta \equiv 0\left(\bmod (1-\zeta)^{2}\right), \beta \not \equiv 0(\bmod 1-\zeta)$, so that

$$
\pm \zeta^{i}-1 \equiv 0\left(\bmod (1-\zeta)^{2}\right) \text {. }
$$

As $i=0,1,2,3,4$ this can only hold with the positive sign and $i=0$, so that $\alpha=\beta$.

3. Dickson's diophantine system. Throughout the rest of this paper $p$ denotes a prime $\equiv 1(\bmod 5)$. Our results involve the diophantine system

$$
\begin{aligned}
16 p & =x^{2}+50 u^{2}+50 v^{2}+125 w^{2}, \quad x \equiv 1(\bmod 5), \\
x w & =v^{2}-4 u v-u^{2} .
\end{aligned}
$$

A theorem of Dickson [1] asserts that (3.1) has exactly four solutions. If $(x, u, v, w)$ is one of these, the other three are given by $(x,-u$, $-v, w),(x, v,-u,-w),(x,-v, u,-w)$. Taking the first equation in (3.1) modulo 8 and the second one modulo 4 we can show (after a little calculation) that $x+2 u-w \equiv x+2 v+w \equiv 0(\bmod 4)$ for any solution of (3.1). This enables us to make the following definition.

Definition 1. For any solution $(x, u, v, w)$ of $?(3.1)$ we define $\psi \equiv \psi(x, u, v, w) \in Z[\zeta]$ by

$$
\psi=c_{1} \zeta+c_{2} \zeta^{2}+c_{3} \zeta^{3}+c_{4} \zeta^{4},
$$

where $c_{i} \equiv c_{i}(x, u, v, w) \in Z(1 \leqq i \leqq 4)$ are given by 


$$
\begin{aligned}
& 4 c_{1}=-x+2 u+4 v+5 w, \\
& 4 c_{2}=-x+4 u-2 v-5 w, \\
& 4 c_{3}=-x-4 u+2 v-5 w, \\
& 4 c_{4}=-x-2 u-4 v+5 w .
\end{aligned}
$$

The properties of $\psi$ that we shall need are given in the next lemma.

Lemma 3. (a) $\psi \bar{\psi}=p$.

(b) $\psi \equiv-1\left(\bmod (1-\zeta)^{2}\right)$.

(c) If $\sigma_{i}(1 \leqq i \leqq 4)$ is the automorphism of $Q(\zeta)$ defined by $\sigma_{i}(\zeta)=\zeta^{i}$ then G.C.D. $\left(\psi_{1}, \psi_{2}\right)$ is a prime of $Z[\zeta]$, where $\psi_{i}=\sigma_{i}(\psi)(1 \leqq i \leqq 4)$.

Proof. (a) As $\zeta+\zeta^{4}=1 / 2(-1+\sqrt{5}), \zeta^{2}+\zeta^{3}=1 / 2(-1-\sqrt{5})$, we have from (3.2)

$$
\begin{aligned}
\psi \bar{\psi}= & \left\{\left(c_{1}^{2}+c_{2}^{2}+c_{3}^{2}+c_{4}^{2}\right)-\frac{1}{2}\left(c_{1} c_{2}+c_{2} c_{3}+c_{3} c_{4}+c_{1} c_{3}\right.\right. \\
& \left.+c_{1} c_{4}+c_{2} c_{4}\right)+\frac{\sqrt{5}}{2}\left(c_{1} c_{2}+c_{2} c_{3}+c_{3} c_{4}-c_{1} c_{3}\right. \\
& \left.\left.-c_{1} c_{4}-c_{2} c_{4}\right)\right\} \\
= & \frac{1}{16}\left(x^{2}+50 u^{2}+50 v^{2}+125 w^{2}\right)-\frac{5 \sqrt{5}}{8} \\
& \times\left(v^{2}-4 u v-u^{2}-x w\right)=p .
\end{aligned}
$$

(b) From (3.1) and (3.3) we have

$$
c_{1}+c_{2}+c_{3}+c_{4}=-x \equiv-1, c_{1}+2 c_{2}+3 c_{3}+4 c_{4} \equiv 0(\bmod 5),
$$

so that $\psi \equiv-1\left(\bmod (1-\zeta)^{2}\right)$.

(c) Let $\pi$ be a prime dividing $p$. As $p \equiv 1(\bmod 5)$ we have $p=\pi_{1} \pi_{2} \pi_{3} \pi_{4}$, where $\pi_{i}=\sigma_{i}(\pi), 1 \leqq i \leqq 4$. By (a) $\psi$ is (up to multiplication by a unit) one of $\pi_{1} \pi_{2}, \pi_{1} \pi_{3}, \pi_{2} \pi_{4}, \pi_{3} \pi_{4}$. In each case G.C.D. $\left(\psi_{1}, \psi_{2}\right)$ is a prime.

Lemma 1 and Lemma 3(c) enable us to define a prime $\mathscr{K}$ of $Z[\zeta]$ as follows.

Definition 2. For any solution $(x, u, v, w)$ of (3.1) we let $\mathscr{K} \equiv \mathscr{K}(x, u, v, w) \in Z[\zeta]$ be such that

$$
\mathscr{K} \sim \text { G.C.D. }\left(\psi_{1}, \psi_{2}\right), \quad \mathscr{K} \equiv-1\left(\bmod (1-\zeta)^{2}\right) .
$$

We remark that $\mathscr{K}$ is not unique, indeed all such $\mathscr{K}$ are given by 
$(-1)^{r}\left(\zeta+\zeta^{4}\right)^{2 r} \mathscr{K}(r \in Z)$. However this does not matter for our purposes. Next we give the prime decomposition of $\psi$ using Lemma 2.

Lemma 4. $\psi=-\mathscr{K}_{1} \mathscr{\varkappa}_{3}$.

Proof. As $\mathscr{K} \sim$ G.C.D. $\left(\psi_{1}, \psi_{2}\right)$ we have $\mathscr{K}_{1} \mid \psi_{1}$, say, $\psi_{1}=\mathscr{K}_{1} \lambda_{1}$. Hence $\psi_{2}=\mathscr{K}_{2} \lambda_{2}$ and as $\mathscr{K}_{1} \mid \psi_{2}$ we must have $\mathscr{K}_{1} \mid \lambda_{2}$, that is $\mathscr{K}_{3} \mid \lambda_{1}$, say $\lambda_{1}=\mathscr{K}_{3} \mu$. Then $\psi_{1}=\mathscr{K}_{1} \mathscr{K}_{3} \mu$ and so we have

$$
\begin{aligned}
\mathscr{K}_{1} \mathscr{K}_{2} \mathscr{K}_{3} \mathscr{K}_{4} & =p=\psi_{1} \bar{\psi}_{1}=\left(\mathscr{K}_{1} \mathscr{K}_{3} \mu\right)\left(\mathscr{K}_{4} \mathscr{K}_{2} \bar{\mu}\right) \\
& =\mathscr{K}_{1} \mathscr{K}_{2} \mathscr{K}_{3} \mathscr{K}_{4} \mu \bar{\mu} .
\end{aligned}
$$

Hence we have $\mu \bar{\mu}=1$, so that $\mu$ is a unit of $Z[\zeta]$, proving that $\psi \sim \mathscr{K}_{1} \mathscr{K}_{3}$. Clearly $\psi$ and $-\mathscr{K}_{1} \mathscr{K}_{3}$ satisfy the conditions of Lemma 2 so that $\psi=-\mathscr{K}_{1} \mathscr{K}_{3}$.

Finally in this section we set for any solution $(x, u, v, w)$ of (3.1):

$$
\begin{aligned}
& \alpha(x, u, v, w) \\
& \quad=\frac{w\left(125 w^{2}-x^{2}\right)+2(x w+5 u v)(25 w-x+20 u-10 v)}{w\left(125 w^{2}-x^{2}\right)+2(x w+5 u v)(25 w-x-20 u+10 v)}
\end{aligned}
$$

and prove

Lemma 5. $\alpha(x, u, v, w) \equiv \zeta(\bmod \mathscr{K})$.

Proof. From (3.2) and $\psi_{1} \equiv \psi_{2} \equiv 0(\bmod \mathscr{K})$ we obtain modulo $\mathscr{K}:$

$$
\begin{aligned}
& 5 c_{1} \equiv\left(\zeta^{2}-1\right) \psi_{3}+(\zeta-1) \psi_{4}, \\
& 5 c_{2} \equiv\left(\zeta^{4}-1\right) \psi_{3}+\left(\zeta^{2}-1\right) \psi_{4}, \\
& 5 c_{3} \equiv(\zeta-1) \psi_{3}+\left(\zeta^{3}-1\right) \psi_{4}, \\
& 5 c_{4} \equiv\left(\zeta^{3}-1\right) \psi_{3}+\left(\zeta^{4}-1\right) \psi_{4} .
\end{aligned}
$$

Appealing to (3.3) we get

$$
\begin{aligned}
x & \equiv \psi_{3}+\psi_{4}, & & 25 u \equiv \alpha \psi_{3}+\beta \psi_{4}, \\
25 v & \equiv \beta \psi_{3}-\alpha \psi_{4}, & & 25 w \equiv-\gamma_{\psi_{3}}+\gamma \psi_{4},
\end{aligned}
$$

where

$$
\begin{aligned}
& \alpha=-2 \zeta+\zeta^{2}-\zeta^{3}+2 \zeta^{4}, \\
& \beta=\zeta+2 \zeta^{2}-2 \zeta^{3}-\zeta^{4}, \\
& \gamma=\zeta-\zeta^{2}-\zeta^{3}+\zeta^{4} .
\end{aligned}
$$

It is easy to check that

$$
\alpha \beta=\alpha^{2}-\beta^{2}=5 \gamma, \quad \gamma^{2}=5 .
$$


After some calculation we find that

$$
\begin{aligned}
& 25\left\{w\left(125 w^{2}-x^{2}\right)+2(x w+5 u v)(25 w-x+20 u-10 v)\right\} \\
& \quad \equiv 4 \psi_{3} \psi_{4}\left((2+2 \zeta) \psi_{3}+2 \zeta^{3} \psi_{4}\right)
\end{aligned}
$$

and

$$
\begin{aligned}
& 25\left\{w\left(125 w^{2}-x^{2}\right)+2(x w+5 u v)(25 w-x-20 u+10 v)\right\} \\
& \equiv 4 \gamma_{3} \psi_{4}\left(\left(2+2 \zeta^{4}\right) \psi_{3}+2 \zeta^{2} \psi_{4}\right)
\end{aligned}
$$

from which the result follows immediately.

4. Outline of method. We start with the necessary and sufficient condition for $D$ (without loss of generality we may take $D$ to be a (positive) prime) to be a quintic residue $(\bmod p)$ in terms of congruences $(\bmod D)$ involving a solution of (3.1). These have been given for $D=2,3,5,7$ in [4] and for $D=11,13,17,19$ in [9]. Results for other values of $D$ could be obtained using the period equation as in [9]. If $D$ is a quintic nonresidue $(\bmod p)$ this condition is used to specify a unique solution of (3.1) by means of congruences $(\bmod D)$. This unique solution is specified in such a way that after using Lemma 4 we find that the corresponding $\mathscr{\varkappa}$ satisfies $(\mathscr{K} / D)_{5}=\zeta$. If $D \neq 5$ we can then appeal to Eisenstein's reciprocity law

"If $\alpha \equiv-1\left(\bmod (1-\zeta)^{2}\right)$ and $a$ is a rational integer prime to 5 then $(\alpha / a)_{5}=(a / \alpha)_{5}$ "

to obtain $(D / \mathscr{K})_{5}=\zeta$, so that $D^{(p-1) / 5} \equiv \alpha(x, u, v, w)(\bmod \mathscr{K})$ by Lemma 5. As both $D^{(p-1) / 5}$ and $\alpha(x, u, v, w)$ are rational we have $D^{(p-1) / 5} \equiv \alpha(x, u, v, w)(\bmod p)$ as required. If $D=5$ we must replace the use of Eisenstein's reciprocity law by Kummer's supplement to the law of quintic reciprocity involving the prime $1-\zeta$ [7]. Unfortunately, this requires working modulo 25 rather than modulo 5 and so involves a large number of cases. We thus give an alternative approach based on a result of Muskat [5].

5. $D=2$. Lehmer [2] has shown that 2 is a quintic residue $(\bmod p)$ if and only if $x \equiv 0(\bmod 2)$, where $(x, u, v, w)$ is any solution of (3.1). Thus if 2 is a quintic nonresidue $(\bmod p)$ we can find by Dickson's theorem a unique solution $(x, u, v, w)$ of (3.1) such that

$$
x \equiv 1(\bmod 2), \quad u \equiv 0(\bmod 2), \quad x+u-v \equiv 0(\bmod 4) .
$$

In terms of this solution a simple calculation using (3.3) shows that $\psi \equiv \zeta^{3}(\bmod 2)$. Then by an examination of cases in conjunction with $\psi=-\mathscr{K}_{1} \mathscr{K}_{3}$ (Lemma 4) we find that

$$
\mathscr{K} \equiv \zeta^{2}, \zeta+\zeta^{3} \text { or } \zeta+\zeta^{2}+\zeta^{3}(\bmod 2),
$$


so that $(\mathscr{K} / 2)_{5}=\zeta$. Appealing to Eisenstein's reciprocity theorem as indicated in $\S 4$ we have reproved

THEOREM 1 (Lehmer [3]). Let $p$ be a prime $\equiv 1(\bmod 5)$ for which 2 is a quintic nonresidue $(\bmod p)$. Let $(x, u, v, w)$ be the unique solution of (3.1) satisfying (5.1). Then we have

$$
2^{(p-1) / 5} \equiv \alpha(x, u, v, w)(\bmod p) .
$$

6. $D=3$. (Lehmer [2] has shown that 3 is a quintic residue $(\bmod p)$ if and only if $u \equiv v \equiv 0(\bmod 3)$, where $(x, u, v, w)$ is any solution of (3.1).) Thus if 3 is a quintic nonresidue $(\bmod p)$ we can find by Dickson's theorem a unique solution $(x, u, v, w)$ of (3.1) satisfying one of

$$
\begin{aligned}
& \text { (a) } x \equiv 1, \quad u \equiv 1, \quad v \equiv 0, \quad w \equiv 2(\bmod 3), \\
& \text { (b) } x \equiv 2, \quad u \equiv 2, \quad v \equiv 0, \quad w \equiv 1(\bmod 3), \\
& \text { (c) } x \equiv 1, \quad u \equiv 2, \quad v \equiv 1, \quad w \equiv 1(\bmod 3), \\
& \text { (d) } x \equiv 2, \quad u \equiv 1, \quad v \equiv 2, \quad w \equiv 2(\bmod 3) .
\end{aligned}
$$

In terms of this solution a simple calculation using (3.3) shows that

$$
\begin{aligned}
& \psi \equiv-\zeta-\zeta^{2}+\zeta^{4}(\bmod 3), \text { if (a) holds , } \\
& \psi \equiv \zeta+\zeta^{2}-\zeta^{4} \quad(\bmod 3), \text { if }(\mathrm{b}) \text { holds , } \\
& \psi \equiv-\zeta^{4} \quad(\bmod 3), \text { if }(\mathrm{c}) \text { holds , } \\
& \psi \equiv \zeta^{4} \quad(\bmod 3), \text { if }(d) \text { holds . }
\end{aligned}
$$

Then by an examination of cases $(\bmod 3)$ in conjunction with Lemma 4 we find that

$$
\begin{aligned}
& \mathscr{K} \equiv \pm\left(\zeta-\zeta^{2}-\zeta^{4}\right), \quad \pm\left(\zeta-\zeta^{2}+\zeta^{3}+\zeta^{4}\right)(\bmod 3), \text { if (a) holds } \\
& \mathscr{K} \equiv \pm\left(\zeta^{3}-\zeta^{4}\right), \quad \pm\left(\zeta-\zeta^{2}-\zeta^{3}\right)(\bmod 3), \text { if }(\mathrm{b}) \text { holds }, \\
& \mathscr{K} \equiv \pm \zeta, \quad \pm\left(\zeta-\zeta^{3}-\zeta^{4}\right)(\bmod 3), \text { if }(\mathrm{c}) \text { holds }, \\
& \mathscr{K} \equiv \pm\left(\zeta^{3}+\zeta^{4}\right), \quad \pm\left(\zeta+\zeta^{3}+\zeta^{4}\right)(\bmod 3), \text { if (d) holds, }
\end{aligned}
$$

so that in every case $(\mathscr{K} / 3)_{5}=\zeta$. Appealing to Eisenstein's reciprocity theorem as before we have the following result.

THEOREM 2 . Let $p$ be a prime $=1(\bmod 5)$ for which 3 is a quintic nonresidue $(\bmod p)$. Let $(x, u, v, w)$ be the unique solution of (3.1) satisfying (6.1). Then we have

$$
3^{(p-1) / 5} \equiv \alpha(x, u, v, w)(\bmod p) .
$$

7. $D=5$. For $p$ a prime $\equiv 1(\bmod 5), g$ a primitive $\operatorname{root}(\bmod p)$, 
$h, k$ integers selected from $0,1,2,3,4$, the cyclotomic number $(h, k)_{5}$ is defined to be the number of solutions $(s, t)$ with $0 \leqq s, t<(p-1) / 5$ of $g^{5 s+h}+1 \equiv g^{5 t+k}(\bmod p)$. Let $(x, u, v, w)$ be any solution of (3.1). Choose $g$ such that $(g / \mathscr{\digamma})_{5}=\zeta$. Then it can be shown that

$$
\begin{aligned}
& 25(0,0)_{5}=p-14+3 x, \\
& 100(0,1)_{5}= 100(1,0)_{5}=100(4,4)_{5}=4 p-16-3 x+50 v+25 w, \\
& 100(0,2)_{5}=100(2,0)_{5}=100(3,3)_{5}=4 p-16-3 x+50 u-25 w, \\
& 100(0,3)_{5}=100(3,0)_{5}=100(2,2)_{5}=4 p-16-3 x-50 u-25 w, \\
& 100(0,4)_{5}=100(4,0)_{5}=100(1,1)_{5}=4 p-16-3 x-50 v+25 w, \\
& 100(1,2)_{5}=100(1,4)_{5}=100(2,1)_{5}=100(3,4)_{5}=100(4,1)_{5} \\
&=100(4,3)_{5}=4 p+4+2 x-50 w, \\
& 100(1,3)_{5}=100(2,3)_{5}=100(2,4)_{5}=100(3,1)_{5}=100(3,2)_{5} \\
&=100(4,2)_{5}=4 p+4+2 x+50 w,
\end{aligned}
$$

and Muskat [5] has shown that

$$
\left.\operatorname{ind}_{g}(5) \equiv(0,4)_{5}-(0,1)_{5}+2\left((0,3)_{5}-(0,2)_{5}\right)\right)(\bmod 5)
$$

so that

$$
\operatorname{ind}_{g}(5) \equiv-2 u-v(\bmod 5) \text {. }
$$

Thus if 5 is a quintic nonresidue $(\bmod p) 2 u+v \not \equiv 0(\bmod 5)$ and by Dickson's theorem there is a unique solution of (3.1) satisfying $2 u+v \equiv 4(\bmod 5)$. With this solution we have $\operatorname{ind}_{g}(5) \equiv 1(\bmod 5)$ and so

$$
5^{(p-1) / 5} \equiv g^{\mathrm{ind}_{g}(5) \cdot(p-1) / 5} \equiv g^{(p-1) / 5} \equiv\left(\frac{g}{\mathscr{K}}\right)_{5} \equiv \zeta(\bmod \mathscr{K}) .
$$

Thus we have proved

THEOREM 3. Let $p$ be a prime $\equiv 1(\bmod 5)$ for which 5 is a quintic nonresidue $(\bmod p)$. Let $(x, u, v, w)$ be the unique solution of (3.1) satisfying $2 u+v \equiv 4(\bmod 5)$. Then we have

$$
5^{(p-1) / 5} \equiv \alpha(x, u, v, w)(\bmod p) .
$$

8. Example. We take $p=311$. A solution of (3.1) in this case is $(-49,7,0,1)$ (see for example [8]) so none of $2,3,5$ is a quintic residue $(\bmod 311)$. The unique solution given by Theorem 1 is $(-49$, $0,7,-1)$ so that

$$
2^{(p-1) / 5}=2^{62} \equiv \frac{2276-98.46}{2276+98.94} \equiv \frac{-2232}{11488} \equiv \frac{-55}{-19} \equiv 52(\bmod 311) .
$$


The unique solution given by Theorem 2 is $(-49,-7,0,1)$ so that

$$
3^{(p-1) / 5}=3^{62} \equiv \frac{-2276+98.66}{-2276-98.214} \equiv \frac{4192}{-23248} \equiv \frac{149}{77} \equiv 216(\bmod 311) .
$$

The unique solution given by Theorem 3 is $(-49,7,0,1)$ so that

$$
5^{(p-1) / 5}=5^{62} \equiv \frac{-2276-98.214}{-2276+98.66} \equiv \frac{-23248}{4192} \equiv \frac{77}{149} \equiv 36(\bmod 311) .
$$

\section{REFERENCES}

1. L. E. Dickson, Cyclotomy, higher congruences, and Waring's problem, Amer. J. Math., 57 (1935), 391-424.

2. Emma Lehmer, The quintic character of 2 and 3, Duke Math. J., 18 (1951), 1118.

3. - On Euler's criterion, J. Austral. Math. Soc., 1 (1959) 64-70.

4. - On the divisors of the discriminant of the period equation, Amer. J. Math., 90 (1968), 375-379.

5. J. B. Muskat, On the solvability of $x^{e} \equiv e(\bmod p)$, Pacific J. Math., 14 (1964), 257-260.

6. K. S. Williams, On Euler's criterion for cubic nonresidues, Proc. Amer. Math. Soc., 49 (1975), 277-283.

7. - Explicit forms of Kummer's complementary theorems to his law of quintic reciprocity, J. für Math., (to appear).

8. - Table of solutions $(x, u, v, w)$ of the diophantine system $16 p=x^{2}+50 u^{2}+$ $50 v^{2}+125 w^{2}, x w=v^{2}-4 u v-u^{2}, x \equiv 1(\bmod 5)$, for primes $p<10,000, p \equiv 1(\bmod 5)$, Unpublished Mathematical Tables File of American Mathematical Society (with B. Lowe).

9. _ Explicit criteria for quintic residuality (submited for publication).

Received June 4, 1974 and in revised form August 8, 1975. Research supported by National Research Council of Canada Grant No. A-7233.

Carleton University 


\section{PACIFIC JOURNAL OF MATHEMATICS}

\section{EDITORS}

RICHARD ARENS (Managing Editor)

University of California

Los Angeles, California 90024

\author{
R. A. Beaumont \\ University of Washington \\ Seattle, Washington 98105
}

\section{J. DugundjI}

Department of Mathematics University of Southern California Los Angeles, California 90007

D. Gilbarg and J. Milgram

Stanford University

Stanford, California 94305

\section{ASSOCIATE EDITORS}
E. F. BECKENBACH
B. H. NeumanN
F. WOLF
K. YoshidA

\section{SUPPORTING INSTITUTIONS}

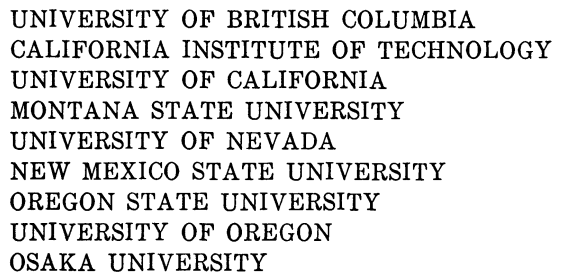

UNIVERSITY OF BRITISH COLUMBIA CALIFORNIA INSTITUTE OF TECHNOLOGY UNIVERSITY OF CALIFORNIA MONTANA STATE UNIVERSITY UNIVERSITY OF NEVADA NEW MEXICO STATE UNIVERSITY OSAKA UNIVERSITY OREGON STATE UNIVERSITY UNIVERSITY OF OREGON

\author{
UNIVERSITY OF SOUTHERN CALIFORNIA \\ STANFORD UNIVERSITY \\ UNIVERSITY OF TOKYO \\ UNIVERSITY OF UTAH \\ WASHINGTON STATE UNIVERSITY \\ UNIVERSITY OF WASHINGTON \\ AMERICAN MATHEMATICAL SOCIETY
}

The Supporting Institutions listed above contribute to the cost of publication of this Journal, but they are not owners or publishers and have no responsibility for its content or policies.

Mathematical papers intended for publication in the Pacific Journal of Mathematics should be in typed form or offset-reproduced, (not dittoed), double spaced with large margins. Please do not use built up fractions in the text of your manuscript. You may however, use them in the displayed equations. Underline Greek letters in red, German in green, and script in blue. The first paragraph or two must be capable of being used separately as a synopsis of the entire paper. Items of the bibliography should not be cited there unless absolutely necessary, in which case they must be identified by author and Journal, rather than by item number. Manuscripts, in triplicate, may be sent to any one of the editors. Please classify according to the scheme of Math. Reviews, Index to Vol. 39. All other communications should be addressed to the managing editor, or Elaine Barth, University of California, Los Angeles, California, 90024.

The Pacific Journal of Mathematics expects the author's institution to pay page charges, and reserves the right to delay publication for nonpayment of charges in case of financial emergency.

100 reprints are provided free for each article, only if page charges have been substantially paid. Additional copies may be obtained at cost in multiples of 50 .

The Pacific Journal of Mathematics is issued monthly as of January 1966. Regular subscription rate: $\$ 72.00$ a year $(6$ Vols., 12 issues). Special rate: $\$ 36.00$ a year to individual members of supporting institutions.

Subscriptions, orders for back numbers, and changes of address should be sent to Pacific Journal of Mathematics, 103 Highland Boulevard, Berkeley, California, 94708.

PUBLISHED BY PACIFIC JOURNAL OF MATHEMATICS, A NON-PROFIT CORPORATION

Printed at Kokusai Bunken Insatsusha (International Academic Printing Co., Ltd.), 8-8, 3-chome, Takadanobaba, Shinjuku-ku, Tokyo 160, Japan. 


\section{Pacific Journal of Mathematics}

\section{Vol. 61, No. 2 December, 1975}

Graham Donald Allen, Francis Joseph Narcowich and James Patrick Williams, An operator version of a theorem of Kolmogorov .......................

Joel Hilary Anderson and Ciprian Foias, Properties which normal operators share with normal derivations and related operators . . . . . . . . . . . . . . . . . . . . .

Constantin Gelu Apostol and Norberto Salinas, Nilpotent approximations and

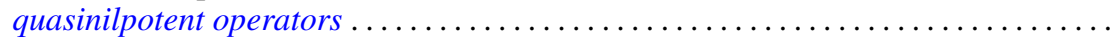

James M. Briggs, Jr., Finitely generated ideals in regular $F$-algebras . . . . . . . . . . .

Frank Benjamin Cannonito and Ronald Wallace Gatterdam, The word problem and power problem in 1-relator groups are primitive recursive ..................

Clifton Earle Corzatt, Permutation polynomials over the rational numbers ...........

L. S. Dube, An inversion of the $S_{2}$ transform for generalized functions . . . . . . . . . . William Richard Emerson, Averaging strongly subadditive set functions in unimodular

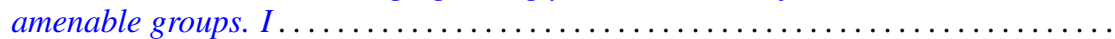

Barry J. Gardner, Semi-simple radical classes of algebras and attainability of

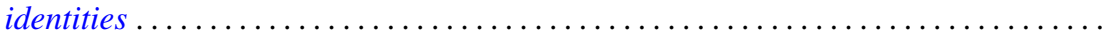

Irving Leonard Glicksberg, Removable discontinuities of A-holomorphic functions ....

Fred Halpern, Transfer theorems for topological structures . . . . . . . . . . . . . . .

H. B. Hamilton, T. E. Nordahl and Takayuki Tamura, Commutative cancellative

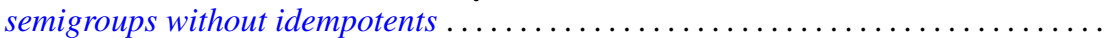

Melvin Hochster, An obstruction to lifting cyclic modules .....................

Alistair H. Lachlan, Theories with a finite number of models in an uncountable power

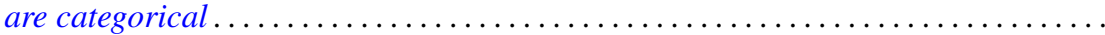

Kjeld Laursen, Continuity of linear maps from $C^{*}$-algebras . . . . . . . . . . . . .

Tsai Sheng Liu, Oscillation of even order differential equations with deviating arguments ....

Jorge Martinez, Doubling chains, singular elements and hyper- $Z$

Mehdi Radjabalipour and Heydar Radjavi, On the geometry of num Thomas I. Seidman, The solution of singular equations, I. Linear equations in Hilbert

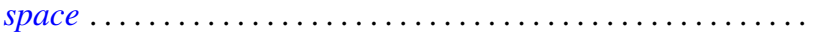

R. James Tomkins, Properties of martingale-like sequences ......

Alfons Van Daele, A Radon Nikodým theorem for weights on von Neumann

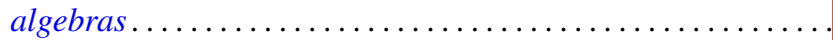

Kenneth S. Williams, On Euler's criterion for quintic nonresidues

Scott Andrew Wolpert, Noncompleteness of the Weil-Petersson metric for Teichmüller

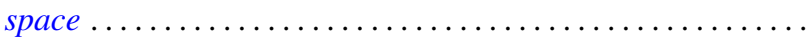

Volker Wrobel, Some generalizations of Schauder's theorem in locally convex

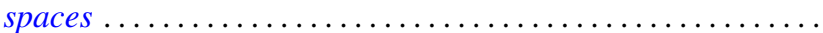

Kelly Denis McKennon, Corrections to: "Multipliers of type $(p, p)$ "; "Multipliers of type $(p, p)$ and multipliers of the group $L_{p}$-algebras"; "Multipliers and the

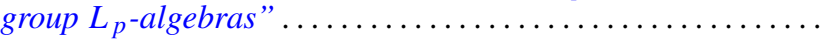

Andrew M. W. Glass, W. Charles (Wilbur) Holland Jr. and Stephen H. McCleary, Correction to: " $a *$-closures to completely distributive lattice-ordered

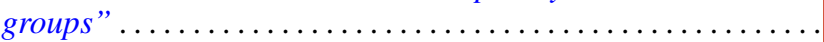

Zvi Arad and George Isaac Glauberman, Correction to: "A characteristic subgroup of

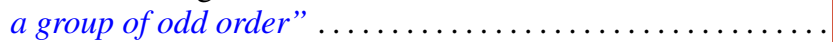

Roger W. Barnard and John Lawson Lewis, Correction to: "Subordination theorems

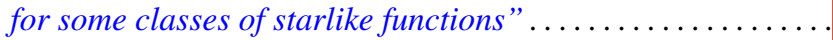

